\title{
An Improved Hybrid Sensor Schedule for Remote State Estimation under Limited Communication Resources
}

\author{
Junfeng Wu, Karl Henrik Johansson and Ling Shi
}

\begin{abstract}
In this paper, we consider remote state estimation. A sensor locally processes its measurement data and sends its local estimate to a remote estimator for further processing. Due to the limited communication resources, the sensor can only communicate with the estimator for a pre-specified number within a given horizon. We propose a hybrid sensor data schedule which introduces an event-triggering mechanism on top of an optimal offline sensor schedule. This hybrid schedule, having a small implementation cost, leads to a smaller estimation error at the remote estimator when compared with the optimal offline sensor schedule.
\end{abstract}

\section{INTRODUCTION}

Advances in modern control, communication and networking technologies enable a new generation of networked control systems (NCSs) [1]. The last decade has witnessed a wealth of NCS applications in smart grid, intelligent transportation systems, health care, environmental monitoring, etc. In many of these applications, remote state estimation is a key component, where sensor data is sent to a remote state estimator over a network. The communication resources for remote estimation, in terms of communication energy and bandwidth, are often scarce. Thus it is of significant importance to understand and obtain a desired tradeoff between the limited communication resources and the remote estimation quality. Such a tradeoff is sometimes possible to achieve via a good sensor scheduling scheme. Most existing sensor schedulers are either offline or online. The recent literature is briefly reviewed below.

Offline Schedulers: Mo et al. [2] proposed a network lifetime maximization policy under an estimation quality constraint. Ambrosino et al. [3] considered remote estimation, where sensors transmit measurements over a shared network to a central base station. Gupta et al. [4] proposed a stochastic sensor selection strategy which minimizes the expected error covariance. Similar approaches can also be found in [5]. Shi et al. [6] considered sensor data scheduling over packet-dropping networks. Due to its limited energy, a sensor has to decide whether to send its local estimate data to the remote estimator at low or high power level at each instance. They showed that the average estimation error is

J. Wu and L. Shi are with the Department of Electronic and Computer Engineering, Hong Kong University of Science and Technology, Clear Water Bay, Kowloon, Hong Kong. Email: jfwu@ust.hk, eesling@ust.hk.

K. H. Johansson is with the ACCESS Linnaeus Center, School of Electrical Engineering, Royal Institute of Technology, Stockholm, Sweden. Email: kallej@ee.kth.se.

The work by J. Wu and L. Shi is supported by a HK RGC CRF grant HKUST11/CRF/10 and a HK RGC GRF grant GRF618612. The work by $\mathrm{K}$. H. Johansson is supported by the Knut and Alice Wallenberg Foundation. minimized when the transmission times at high power mode are separated as much as possible.

Online Schedulers: Åström and Bernhardsson [7] considered a simple first-order stochastic system. They showed that at the same average sampling rate of a periodic sampler, the event-based sampler leads to smaller state variance. Imer and Başar [8] considered an estimation problem over a scalar linear system with a limited number of observations. Upon observing the process, the observer needs to make a decision whether to send some observation information to the estimator. Li et al. [9] extended the results of [8] to vector linear systems. A sub-optimal event-trigger is given to minimize the mean square estimation error through a computationally efficient way. Cogill [10] considered the problem of controlling a system with limited actuation and sampling rate, where a control action is only applied when a certain event occurs. An event-based control policy was proposed which minimizes the upper bound on control performance using a quadratic approximate value function.

Offline schedules are often easier to compute then online schedules, but at the same time, may have worse performance. To make the best use of each approach, Shi et al. [11] proposed a novel hybrid sensor schedule which introduced an event-triggering mechanism on top of an optimal offline schedule. They considered a scenario when a sensor can only communicate with a remote state estimator $m$ times within a time-horizon $T \gg m$. By selecting the event-triggering threshold $\delta>0$ appropriately, the hybrid sensor schedule was shown to have better performance than the optimal offline one. However, the results of [11] have the following limitations:

1) When an event occurs, the error covariance matrix is not explicitly given, but only an upper bound.

2) The critical threshold $\delta_{\max }$ below which the hybrid schedule outperforms the optimal offline one is not explicitly given, but only a lower bound.

3) Selection of the optimal threshold $\delta$ relies on Monte Carlo simulations.

This paper presents an improved hybrid sensor schedule based on the results of [11]. The main contributions are summarized as follows.

1) A modified event-triggering mechanism is given under which a closed-form expression on the estimation error covariance when an event happens is derived.

2) A closed-form expression on the critical threshold $\delta_{\max }$ is given.

3) The optimal threshold $\delta$ is given analytically for first- 


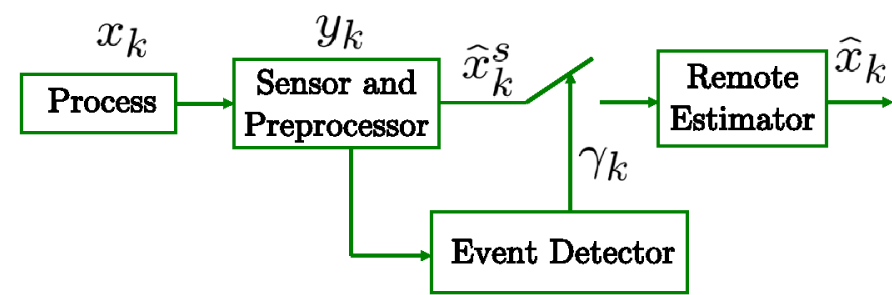

Fig. 1. Hybrid scheduling architecture for remote estimation.

order systems and can be solved efficiently for higherorder systems.

The remainder of the paper is organized as follows. Mathematical models of the system are given in Section II. Section III reviews some key results of [11]. A hybrid sensor schedule is introduced in Section IV and analyzed in Section V. Section VI demonstrates the main theoretical results. Some concluding remarks are given in the end.

Notations: $\mathbb{Z}$ is the set of non-negative integers. $\mathbb{N}$ is the set of positive integers. $k \in \mathbb{Z}$ is the time index. $\mathbb{R}^{n}$ is the $n$ dimensional Euclidean space. $\mathbb{S}_{+}^{n}$ is the set of $n$ by $n$ positive semi-definite matrices. When $X \in \mathbb{S}_{+}^{n}$, it is written as $X \geq 0 . X \geq Y$ if $X-Y \in \mathbb{S}_{+}^{n} . \mathbb{E}[\cdot]$ is the expectation of a random variable and $\mathbb{E}[\cdot \mid \cdot]$ is the conditional expectation. $\operatorname{Pr}(\cdot)$ is the probability of a random event. $\operatorname{Tr}(\cdot)$ is the trace of a matrix and $\|\cdot\|_{\infty}$ is the infinity norm of a vector. For functions $f, f_{1}, f_{2}: \mathbb{S}_{+}^{n} \rightarrow \mathbb{S}_{+}^{n}, f_{1} \circ f_{2}$ is defined as $f_{1} \circ f_{2}(X) \triangleq f_{1}\left(f_{2}(X)\right)$ and $f^{t}$ is defined as $f^{t}(X) \triangleq \underbrace{f \circ f \circ \cdots \circ f}_{t \text { times }}(X)$.

\section{PROBLEM SETUP}

Consider the following system (Fig. 1)

$$
\begin{aligned}
x_{k+1} & =A x_{k}+w_{k}, \\
y_{k} & =C x_{k}+v_{k},
\end{aligned}
$$

where $x_{k} \in \mathbb{R}^{n}$ is the process state vector, $y_{k} \in \mathbb{R}^{m}$ is the observation vector, $w_{k} \in \mathbb{R}^{n}$ and $v_{k} \in \mathbb{R}^{m}$ are zeromean Gaussian random vectors with $\mathbb{E}\left[w_{k} w_{j}{ }^{\prime}\right]=\delta_{k j} Q(Q \geq$ $0), \mathbb{E}\left[v_{k} v_{j}{ }^{\prime}\right]=\delta_{k j} R(R>0), \mathbb{E}\left[w_{k} v_{j}{ }^{\prime}\right]=0 \forall j, k$. The initial state $x_{0}$ is a zero-mean Gaussian random vector that is uncorrelated with $w_{k}$ and $v_{k}$ and has covariance $\Pi_{0} \geq 0$. The pair $(A, C)$ is assumed to be observable and $(A, \sqrt{Q})$ is controllable.

Assume the sensor runs a Kalman filter to compute

$$
\hat{x}_{k}^{s}=\mathbb{E}\left[x_{k} \mid y_{0}, \ldots, y_{k}\right],
$$

the local MMSE estimate of $x_{k}$ in (1). The estimation error $e_{k}^{s}$ and error covariance matrix $P_{k}^{s}$ are defined as follows:

$$
\begin{aligned}
e_{k}^{s} & \triangleq x_{k}-\hat{x}_{k}^{s}, \\
P_{k}^{s} & \triangleq \mathbb{E}\left[\left(e_{k}^{s}\right)\left(e_{k}^{s}\right)^{\prime} \mid y_{0}, \ldots, y_{k}\right] .
\end{aligned}
$$

After $\hat{x}_{k}^{s}$ is obtained, the sensor decides whether to send it to the remote estimator. Let $\gamma_{k}$ be the decision variable at time $k$, i.e., if $\gamma_{k}=1, \hat{x}_{k}^{s}$ is sent, otherwise $\hat{x}_{k}^{s}$ is not sent.

Let $T \in \mathbb{N}$ be the time-horizon and define a schedule $\theta$ as

$$
\theta=\left\{\gamma_{1}, \ldots, \gamma_{T}\right\} \in\{0,1\}^{T} .
$$

Under a given $\theta$, the remote estimator calculates $\hat{x}_{k}$ and $P_{k}$, its own MMSE estimate of $x_{k}$ and the associated error covariance. Define $J(\theta)$ as the trace of the average expected estimation error covariance, i.e.,

$$
J(\theta) \triangleq \frac{1}{T} \sum_{k=1}^{T} \operatorname{Tr}\left(\mathbb{E}\left[P_{k}(\theta)\right]\right) .
$$

Consider the following problem from [11]:

Problem 2.1:

$$
\begin{array}{ll}
\min _{\theta} & J(\theta), \\
\text { s.t. } & \sum_{k=1}^{T} \gamma_{k}(\theta)=m .
\end{array}
$$

\section{PRELIMINARIES}

\section{A. Kalman Filter Preliminaries}

It is well known that $\hat{x}_{k}^{s}$ and $P_{k}^{s}$ can be computed through a Kalman filter as follows:

$$
\begin{aligned}
\hat{x}_{k \mid k-1}^{s} & =A \hat{x}_{k-1}^{s}, \\
P_{k \mid k-1}^{s} & =A P_{k-1}^{s} A^{\prime}+Q, \\
K_{k} & =P_{k \mid k-1}^{s} C^{\prime}\left[C P_{k \mid k-1}^{s} C^{\prime}+R\right]^{-1}, \\
\hat{x}_{k}^{s} & =A \hat{x}_{k-1}^{s}+K_{k}\left(y_{k}-C A \hat{x}_{k-1}^{s}\right), \\
P_{k}^{s} & =\left(I-K_{k} C\right) P_{k \mid k-1}^{s},
\end{aligned}
$$

where the recursion starts from $\hat{x}_{0}^{s}=0$ and $P_{0}^{s}=\Pi_{0}$. At the estimator side, it is straightforward to show that the optimal state estimate $\hat{x}_{k}$ is given by

$$
\hat{x}_{k}= \begin{cases}A \hat{x}_{k-1}, & \text { if } \gamma_{k}=0, \\ \hat{x}_{k}^{s}, & \text { if } \gamma_{k}=1 .\end{cases}
$$

For simplicity, define functions $h$ and $\tilde{g}: \mathbb{S}_{+}^{n} \rightarrow \mathbb{S}_{+}^{n}$ as

$$
\begin{gathered}
h(X) \triangleq A X A^{\prime}+Q \\
\tilde{g}(X) \triangleq X-X C^{\prime}\left[C X C^{\prime}+R\right]^{-1} C X .
\end{gathered}
$$

Similar to [11], we assume the Kalman filter has entered steady state to simplify the discussion, i.e.,

$$
P_{k}^{s}=\bar{P}, k \geq 1
$$

where $\bar{P}$ is the steady-state error covariance. As the unique positive semi-definite solution [12] of $\tilde{g} \circ h(X)=X, \bar{P}$ has the following property.

Lemma 3.1: For $0 \leq t_{1} \leq t_{2}$, the following inequality holds:

$$
h^{t_{1}}(\bar{P}) \leq h^{t_{2}}(\bar{P})
$$

In addition, if $t_{1}<t_{2}$, then

$$
\operatorname{Tr}\left(h^{t_{1}}(\bar{P})\right)<\operatorname{Tr}\left(h^{t_{2}}(\bar{P})\right) .
$$

Proof: See [11]. 


\section{B. Optimal Offline Sensor Schedule}

Shi et al. [11] introduced the optimal offline sensor schedule to Problem 2.1. For simplicity, they considered $m=2 t-$ 1 and $T=4 q t-1$ for $t, q \in \mathbb{N}$. Other forms of $T$ and $m$ can be dealt similarly. As shown in the following proposition, the optimal offline schedule is a periodic schedule with period $2 q$ and the $m$ communication times between the sensor and the remote estimator are separated as uniform as possible.

Proposition 3.2: The optimal offline schedule $\theta_{\text {off }}^{\star} \in$ $\{0,1\}^{T}$ that minimizes $J(\theta)$ in (5) is given by:

$$
\gamma_{2 l q}=1 \forall l=1, \ldots, 2 t-1 \text {, and } \gamma_{k}=0 \text { otherwise. }
$$

Under $\theta_{\text {off }}^{\star}, P_{k}$ evolves as

$$
P_{k}= \begin{cases}h\left(P_{k-1}\right), & \text { if } \gamma_{k}=0, \\ \bar{P}, & \text { if } \gamma_{k}=1 .\end{cases}
$$

The corresponding minimum $J(\theta)$ is given by

$$
J\left(\theta_{\text {off }}^{\star}\right)=\frac{2 t}{T} \sum_{i=0}^{2 q-1} \operatorname{Tr}\left(h^{i}(\bar{P})\right)-\frac{1}{T} \operatorname{Tr}(\bar{P}) .
$$

Proof: See [11].

\section{A Hybrid SENSOR SCHEdule}

In this section, we consider the hybrid schedule proposed in [11]. We assume $m=2 t-1$ and $T=4 q t-1$ for $t, q \in \mathbb{N}$. The lemmas in this section, if without proof, follow directly from [11].

\section{A. A Hybrid Sensor Schedule}

First note that the sensor is able to calculate $\hat{x}_{k}$ as it has access to all $\gamma_{k}$ 's. Define $\varepsilon_{k}$ as

$$
\varepsilon_{k} \triangleq \hat{x}_{k}^{s}-A \hat{x}_{k-1},
$$

where $A \hat{x}_{k-1}$ is the predicted state estimate at the estimator based on the previous optimal state estimate $\hat{x}_{k-1}$. Thus if $\hat{x}_{k}^{s}$ is not sent at time $k, \varepsilon_{k}$ will indicate how close is the state estimate at the estimator from the optimal state estimate at the sensor.

Lemma 4.1: The following statements on $e_{k}^{s}$ hold:

1) $e_{k}^{s}$ is independent of $\hat{x}_{k}^{s}$, hence $\mathbb{E}\left[\left(e_{k}^{s}\right)\left(\hat{x}_{k}^{s}\right)^{\prime}\right]=0$.

2) $e_{k}^{s}$ is zero-mean Gaussian.

3) $e_{k}^{s}$ is independent of $A^{d} \hat{x}_{k-d}^{s}$ and $\hat{x}_{k}^{s}-A^{d} \hat{x}_{k-d}^{s}$ for any $d \in \mathbb{N}$.

Lemma 4.2: $e_{k}^{s}$ and $\varepsilon_{k}$ are independent and $\mathbb{E}\left[e_{k}^{s} \varepsilon_{k}^{\prime}\right]=0$.

Lemma 4.3: $\varepsilon_{2 l q}$ is zero-mean Gaussian and its covariance is independent of $l$.

Lemma 4.4: $\mathbb{E}\left[\varepsilon_{2 l q} \varepsilon_{2 l q}^{\prime}\right]=h^{2 q}(\bar{P})-\bar{P}$.

Let the rank of $h^{2 q}(P)-\bar{P}$ be $r$. From Lemma 3.1, $h^{2 q}(\bar{P})-\bar{P} \geq 0$, hence there exists an orthonormal matrix $U \in \mathbb{R}^{n \times n}$ such that

$$
U^{\prime}\left(h^{2 q}(\bar{P})-\bar{P}\right) U=\left[\begin{array}{ll}
\Lambda & 0 \\
0 & 0
\end{array}\right],
$$

where $\Lambda=\operatorname{diag}\left(\lambda_{1}, \ldots, \lambda_{\mathrm{r}}\right)$ and $\lambda_{1}, \ldots, \lambda_{r} \in \mathbb{R}$ are the $r$ nonzero eigenvalues of $h^{2 q}(\bar{P})-\bar{P}$. Define $F \in \mathbb{R}^{n \times n}$ as:

$$
F \triangleq U\left[\begin{array}{ll}
\Lambda^{-\frac{1}{2}} & 0 \\
0 & I_{n-r}
\end{array}\right] \text {. }
$$

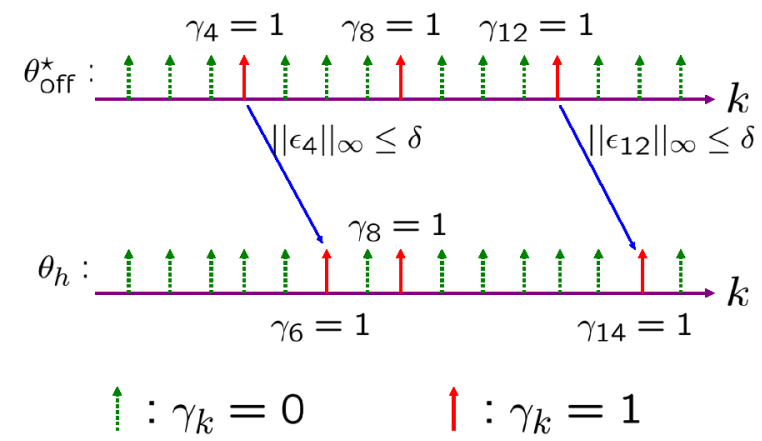

Fig. 2. Realization of $\theta_{\mathrm{off}}^{\star}$ and $\theta_{h}$

Then

$$
F^{\prime}\left(h^{2 q}(\bar{P})-\bar{P}\right) F=\left[\begin{array}{ll}
I_{r} & 0 \\
0 & 0
\end{array}\right] .
$$

Define $\epsilon_{2 l q}$ as $\epsilon_{2 l q} \triangleq F^{\prime} \varepsilon_{2 l q}$. We propose the hybrid schedule $\theta_{\mathrm{h}}$ as follows: for a given threshold $\delta>0, \theta_{\mathrm{h}}$ is identical to $\theta_{\text {off }}^{\star}$ except at $k=2 l q$ when $l$ is odd, in which instances, if $\left\|\epsilon_{2 l q}\right\|_{\infty} \leq \delta$, set $\gamma_{2 l q}=0$ and $\gamma_{(2 l+1) q}=1$.

Since $w_{k}$ 's and $v_{k}$ 's are random, the instances for the sensor to send $\hat{x}_{k}^{s}$ to the remote estimator under $\theta_{h}$ also become random. On the contrary, under $\theta_{\text {off }}^{\star}$, the communication instances are fixed a priori. Fig. 2 shows a sample realization of the sensor communication instances under both $\theta_{\text {off }}^{\star}$ and $\theta_{h}$ for $t=2, q=2, m=3$ and $T=15$.

Remark 4.5: In [11], $\left|\varepsilon_{2 l q}\right| \leq \delta$ was used to define the event, i.e., when $l$ is odd, if $\left|\varepsilon_{2 l q}\right| \leq \delta$, then $\gamma_{2 l q}=0$. Since the entries of $\varepsilon_{2 l q}$ might be mutually correlated, it is difficult to analyze the estimation quality under the hybrid sensor schedule. For example, when an event is triggered, no closed-form but only an upper bound of $P_{2 l q}$ could be obtained, and consequently, selecting the optimal threshold $\delta$ has to rely on Monte Carlo simulations. In this paper, through a linear transformation $F^{\prime}, \varepsilon_{2 l q}$ is changed to $\epsilon_{2 l q}$, a random vector with mutually independent zero-mean Gaussian entries, which makes the analysis considerably simpler.

Lemma 4.6:

$$
\begin{aligned}
p_{\delta} \triangleq \operatorname{Pr}\left(\left\|\epsilon_{2 l q}\right\|_{\infty}>\delta\right) & =1-[1-2 Q(\delta)]^{r}, \\
\mathbb{E}\left[\varepsilon_{2 l q} \varepsilon_{2 l q}^{\prime} \mid\left\|\epsilon_{2 l q}\right\|_{\infty} \leq \delta\right] & =[1-\beta(\delta)]\left[h^{2 q}(\bar{P})-\bar{P}\right],
\end{aligned}
$$

where $Q(\delta)$ is the $Q$-function defined by

$$
Q(\delta) \triangleq \int_{\delta}^{+\infty} \frac{1}{\sqrt{2 \pi}} e^{-\frac{x^{2}}{2}} \mathrm{~d} x
$$

and

$$
\beta(\delta) \triangleq \frac{2}{\sqrt{2 \pi}} \delta e^{-\frac{\delta^{2}}{2}}[1-2 Q(\delta)]^{-1} .
$$

Proof: Let $\epsilon_{2 l q}=\left[\begin{array}{l}\xi_{2 l q}^{1} \\ \xi_{2 l q}^{2}\end{array}\right]$ where $\xi_{2 l q}^{1} \in \mathbb{R}^{r}$ and $\xi_{2 l q}^{2} \in$ $\mathbb{R}^{n-r}$. Since $\mathbb{E}\left[\varepsilon_{2 l q} \varepsilon_{2 l q}^{\prime}\right]=h^{2 q}(\bar{P})-\bar{P}$, we have

$$
\mathbb{E}\left[\epsilon_{2 l q} \epsilon_{2 l q}^{\prime}\right]=F^{\prime} \mathbb{E}\left[\varepsilon_{2 l q} \varepsilon_{2 l q}^{\prime}\right] F=\left[\begin{array}{ll}
I_{r} & 0 \\
0 & 0
\end{array}\right] .
$$


Hence $\xi_{2 l q}^{1}$ is a zero mean Gaussian multivariate random variable with unit variance, and $\xi_{2 l q}^{2}=0$ almost surely. Lemma 1.2 leads to the following:

$\left.1-p_{\delta}=\operatorname{Pr}\left(\left\|\epsilon_{2 l q}\right\|_{\infty} \leq \delta\right)=\operatorname{Pr}\left(\left\|\xi_{k}^{1}\right\|_{\infty} \leq \delta\right)\right)=[1-2 Q(\delta)]^{r}$,

which shows (20), and

$$
\mathbb{E}\left[\epsilon_{2 l q} \epsilon_{2 l q}^{\prime} \mid\left\|\epsilon_{2 l q}\right\|_{\infty} \leq \delta\right]=[1-\beta(\delta)]\left[\begin{array}{ll}
I_{r} & 0 \\
0 & 0
\end{array}\right] .
$$

Since $U^{\prime}=U^{-1}$, one has

$$
\begin{aligned}
& \mathbb{E}\left[\varepsilon_{2 l q} \varepsilon_{2 l q}^{\prime} \mid\left\|\epsilon_{2 l q}\right\|_{\infty} \leq \delta\right] \\
= & \left(F^{\prime}\right)^{-1} \mathbb{E}\left[\epsilon_{2 l q} \epsilon_{2 l q}^{\prime} \mid\left\|\epsilon_{2 l q}\right\|_{\infty} \leq \delta\right] F^{-1} \\
= & {[1-\beta(\delta)] U\left[\begin{array}{cc}
\Lambda & 0 \\
0 & 0
\end{array}\right] U^{\prime} } \\
= & {[1-\beta(\delta)]\left[h^{2 q}(\bar{P})-\bar{P}\right], }
\end{aligned}
$$

which completes the proof.

Corollary 4.7: $\gamma_{2 l q}$ 's are i.i.d Bernoulli random variables with $\operatorname{Pr}\left(\gamma_{2 l q}=0\right)=1-p_{\delta}$, and $\operatorname{Pr}\left(\gamma_{2 l q}=1\right)=p_{\delta}$.

Proof: Note that $\operatorname{Pr}\left(\gamma_{2 l q}=0\right)=\operatorname{Pr}\left(\left\|\epsilon_{2 l q}\right\|_{\infty} \leq \delta\right)$. The assertion is a direct result from (20).

We now introduce one of the main results of this paper, in which a closed-form expression on the error covariance matrix $P_{k}$ is obtained. by

Theorem 4.8: The error covariance $P_{k}$ under $\theta_{\mathrm{h}}$ is given

$$
P_{k}= \begin{cases}h\left(P_{k-1}\right), & \text { if } \gamma_{k}=0, \\ \bar{P}, & \text { if } \gamma_{k}=1,\end{cases}
$$

except at those time instances $k=2 l q$ when $l$ is odd and $\left\|\epsilon_{2 l q}\right\|_{\infty} \leq \delta$, in which cases, $\gamma_{2 l q}=0$ and $P_{2 l q}$ is given by

$$
P_{2 l q}=\bar{P}+[1-\beta(\delta)]\left[h^{2 q}(\bar{P})-\bar{P}\right] .
$$

Proof: We only prove the exceptional case as the other cases are straightforward. At the estimator, if no packet is received at time $k=2 l q$, then $\gamma_{2 l q}=0$, which also implies $\left\|\epsilon_{2 l q}\right\|_{\infty} \leq \delta$. Therefore

$$
\begin{aligned}
P_{2 l q} & =\mathbb{E}\left[\left(x_{2 l q}-\hat{x}_{2 l q}\right)(\cdot)^{\prime} \mid\left\|\epsilon_{2 l q}\right\|_{\infty} \leq \delta\right] \\
& =\mathbb{E}\left[\left(x_{2 l q}-\hat{x}_{2 l q}^{s}+\hat{x}_{2 l q}^{s}-\hat{x}_{2 l q}\right)(\cdot)^{\prime} \mid\left\|\epsilon_{2 l q}\right\|_{\infty} \leq \delta\right] \\
& =\mathbb{E}\left[\left(e_{2 l q}^{s}+\varepsilon_{2 l q}\right)\left(e_{2 l q}^{s}+\varepsilon_{2 l q}\right)^{\prime}\left\|\epsilon_{2 l q}\right\|_{\infty} \leq \delta\right] \\
& =\mathbb{E}\left[\left(e_{2 l q}^{s}\right)\left(e_{2 l q}^{s}\right)^{\prime} \mid\left\|\epsilon_{2 l q}\right\|_{\infty} \leq \delta\right]+ \\
& \mathbb{E}\left[\varepsilon_{2 l q} \varepsilon_{2 l q}^{\prime} \mid\left\|\epsilon_{2 l q}\right\|_{\infty} \leq \delta\right] \\
= & \bar{P}+[1-\beta(\delta)]\left[h^{2 q}(\bar{P})-\bar{P}\right],
\end{aligned}
$$

where the second last equality is from Lemma 4.2 and the last equality is from Lemma 4.6.

\section{Performance Analysis}

In this section we compare the performance of $\theta_{\mathrm{h}}$ with that of $\theta_{\text {off }}^{\star}$. The following lemma on properties of $\beta(\delta)$ in (21) is useful to derive the main result. The proof is straightforward and is omitted.

Lemma 5.1: 1) $\beta(\delta)>1-\delta^{2}$.

2) $\beta(\delta)$ is strictly decreasing in $\delta$.
3) $\lim _{\delta \rightarrow 0} \beta(\delta)=1$.

4) $\lim _{\delta \rightarrow+\infty} \beta(\delta)=0$.

The following result provides a sufficient and necessary condition on $\delta$ such that $\theta_{\mathrm{h}}$ outperforms $\theta_{\text {off }}^{\star}$.

Theorem 5.2: The following statements hold.

1) $J\left(\theta_{\mathrm{h}}\right) \leq J\left(\theta_{\text {off }}^{\star}\right)$ iff $\delta \in\left[0, \delta_{\max }\right]$, where $\delta_{\max }$ is the unique solution to

$$
\beta(\delta)\left(\Gamma_{2}-\Gamma_{0}\right)=\Gamma_{2}-\Gamma_{1}
$$

and

$$
\Gamma_{j}=\operatorname{Tr}\left[\sum_{i=j q}^{(j+1) q-1} h^{i}(\bar{P})\right], j=0,1,2 .
$$

2) For any $\delta \in\left(0, \delta_{\max }\right)$ and for any realization $\phi$ of $\theta_{\mathrm{h}}$,

$$
J(\phi) \leq J\left(\theta_{\text {off }}^{\star}\right) .
$$

Furthermore there exists a positive probability of $\phi$ such that (24) becomes strict, hence $J\left(\theta_{\mathrm{h}}\right)<J\left(\theta_{\text {off }}^{\star}\right)$.

Proof: (1) Recall that $p_{\delta}=\operatorname{Pr}\left(\left\|\epsilon_{2 l q}\right\|_{\infty}>\delta\right)$. For any odd number $l$,

$$
\begin{aligned}
& \sum_{k=2(l-1) q}^{2(l+1) q-1} \mathbb{E}\left[P_{k}\left(\theta_{\mathrm{h}}\right)\right] \\
= & \sum_{i=0}^{2 q-1} h^{i}(\bar{P})+p_{\delta} \sum_{i=0}^{2 q-1} h^{i}(\bar{P})+\left(1-p_{\delta}\right)\left[\beta(\delta) \sum_{i=0}^{q-1} h^{i}(\bar{P})\right. \\
& \left.+[1-\beta(\delta)] \sum_{i=2 q}^{3 q-1} h^{i}(\bar{P})+\sum_{i=0}^{q-1} h^{i}(\bar{P})\right],
\end{aligned}
$$

which is independent of $l$. Define $D_{\delta}$ as

$$
\begin{aligned}
& D_{\delta} \\
\triangleq & \operatorname{Tr}\left[\sum_{i=q}^{2 q-1} h^{i}(\bar{P})-\beta(\delta) \sum_{i=0}^{q-1} h^{i}(\bar{P})-[1-\beta(\delta)] \sum_{i=2 q}^{3 q-1} h^{i}(\bar{P})\right] \\
= & \Gamma_{1}-\beta(\delta) \Gamma_{0}-[1-\beta(\delta)] \Gamma_{2} .
\end{aligned}
$$

With some manipulation, we obtain

$$
J\left(\theta_{\text {off }}^{\star}\right)-J\left(\theta_{\mathrm{h}}\right)=\frac{1}{4 q}\left(1-p_{\delta}\right) D_{\delta} .
$$

Since $1-p_{\delta} \geq 0, J\left(\theta_{\text {off }}^{\star}\right) \geq J\left(\theta_{\mathrm{h}}\right)$ iff $D_{\delta} \geq 0$. One can easily verify that $\Gamma_{2}>\Gamma_{1}>\Gamma_{0}$. From Lemmas 3.1 and 5.1, $D_{\delta}$ is strictly decreasing in $\delta$ and

$$
D_{0}=\Gamma_{1}-\Gamma_{0}>0, \quad D_{\infty}=\Gamma_{1}-\Gamma_{2}<0 .
$$

Hence there is a unique $\delta_{\max }$ such that $D_{\delta_{\max }}=0$, which corresponds to the $\delta_{\max }$ in (23). One notes that $\left(\Gamma_{2}-\Gamma_{1}\right) /\left(\Gamma_{2}-\Gamma_{0}\right) \in(0,1)$, thus (23) must have a solution. Furthermore, for all $\delta<\delta_{\max }, D_{\delta}>0$ and for all $\delta>\delta_{\max }, D_{\delta}<0$.

(2) Let $\delta_{\max }>0$. For any $\delta \in\left(0, \delta_{\max }\right)$ and for any realization $\phi$ of $\theta_{\mathrm{h}}$, if $\left\|\epsilon_{2 l q}\right\|_{\infty}>\delta$ for all odd number $l$, then $\phi$ is the same as $\theta_{\text {off }}^{\star}$. Hence $J(\phi)=J\left(\theta_{\text {off }}^{\star}\right)$. Otherwise if there exists an odd number $l$ such that $\left\|\epsilon_{2 l q}\right\|_{\infty} \leq \delta$, then similar to the proof of the first statement, one easily verifies 
that $J(\phi)<J\left(\theta_{\text {off }}^{\star}\right)$. Notice that the probability of those $\phi$ 's with at least one $l$ such that $\left\|\epsilon_{2 l q}\right\|_{\infty} \leq \delta$ is positive, hence

$J\left(\theta_{\mathrm{h}}\right)=\sum_{\phi} \operatorname{Pr}(\phi) J(\phi)<\sum_{\phi} \operatorname{Pr}(\phi) J\left(\theta_{\text {off }}^{\star}\right)=J\left(\theta_{\text {off }}^{\star}\right)$.

Remark 5.3: Since $\beta(\delta)$ is monotonically decreasing in $\delta$ from Lemma 5.1 and $\Gamma_{0}, \Gamma_{1}, \Gamma_{2}$ can be easily computed, (23) can be solved efficiently via Newton's method.

To minimize $J\left(\theta_{\mathrm{h}}\right)$, we simply compute the $\delta$ that maximizes $\left(1-p_{\delta}\right) D_{\delta}$ so that the difference between $J\left(\theta_{\mathrm{h}}\right)$ and $J\left(\theta_{\text {off }}^{\star}\right)$ is maximum. Let $\delta^{\star}$ be the optimal $\delta$, i.e.,

$$
\delta^{\star} \triangleq \arg \min _{\delta>0} J\left(\theta_{\mathrm{h}}\right)=\arg \max _{\delta>0}[1-2 Q(\delta)]^{r} D_{\delta} .
$$

The optimal $\delta^{\star}$ for the schedule $\theta_{\mathrm{h}}$ is presented in the following theorem.

Theorem 5.4: The optimal $\delta^{\star}$ is the unique solution to

$$
\delta^{2}+(r-1)[1-\beta(\delta)]-r \frac{\Gamma_{1}-\Gamma_{0}}{\Gamma_{2}-\Gamma_{0}}=0 .
$$

In particular, if $r=1$,

$$
\delta^{\star}=\sqrt{\frac{\Gamma_{1}-\Gamma_{0}}{\Gamma_{2}-\Gamma_{0}}} .
$$

Proof: (1) When $r=1$,

$$
\frac{\mathrm{d}\left\{[1-2 Q(\delta)] D_{\delta}\right\}}{\mathrm{d} \delta}=\frac{2 e^{-\frac{\delta^{2}}{2}}}{\sqrt{2 \pi}}\left[\Gamma_{1}-\left(1-\delta^{2}\right) \Gamma_{0}-\delta^{2} \Gamma_{2}\right],
$$

which is an increasing function. Setting (28) to zero, one obtains (27).

(2) When $r \geq 2$,

$$
\begin{aligned}
& \frac{\mathrm{d}\left\{[1-2 Q(\delta)]^{r} D_{\delta}\right\}}{\mathrm{d} \delta} \\
= & \frac{2 e^{-\frac{\delta^{2}}{2}}}{\sqrt{2 \pi}}[1-2 Q(\delta)]^{r-2}\left\{[1-2 Q(\delta)]\left(\Gamma_{2}-\Gamma_{0}\right)\left(1-\delta^{2}\right)\right. \\
& \left.+\left(\Gamma_{2}-\Gamma_{0}\right)(r-1) \frac{2 \delta e^{-\frac{\delta^{2}}{2}}}{\sqrt{2 \pi}}-[1-2 Q(\delta)]\left(\Gamma_{2}-\Gamma_{1}\right) r\right\} \\
= & \frac{2 e^{-\frac{\delta^{2}}{2}}}{\sqrt{2 \pi}}[1-2 Q(\delta)]^{r-1}\left\{\left(\Gamma_{2}-\Gamma_{0}\right)\left[1-\delta^{2}+(r-1) \beta(\delta)\right]\right. \\
& \left.-\left(\Gamma_{2}-\Gamma_{1}\right) r\right\} .
\end{aligned}
$$

Define

$$
\varphi(\delta) \triangleq\left(\Gamma_{2}-\Gamma_{0}\right)\left[1-\delta^{2}+(r-1) \beta(\delta)\right]-\left(\Gamma_{2}-\Gamma_{1}\right) r .
$$

From Lemma 5.1, $\varphi(\delta)$ is strictly decreasing in $\delta$ with

$$
\lim _{\delta \rightarrow 0} \varphi(\delta)=r
$$

and

$$
\begin{aligned}
\varphi\left(\delta_{\max }\right) & <\left(\Gamma_{2}-\Gamma_{0}\right)\left[\beta\left(\delta_{\max }\right)+(r-1) \beta\left(\delta_{\max }\right)\right]-\left(\Gamma_{2}-\Gamma_{1}\right) r \\
& =0 .
\end{aligned}
$$

Note that $\frac{2 e^{-\frac{\delta^{2}}{2}}}{\sqrt{2 \pi}}[1-2 Q(\delta)]^{r-1}>0$, therefore, the optimal $\delta^{\star}$ exists and is the unique solution to (26).

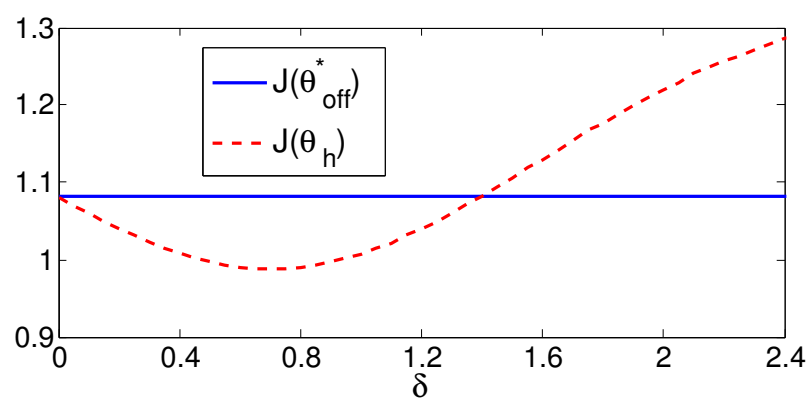

Fig. 3. Comparison of $\theta_{\mathrm{h}}$ and $\theta_{\text {off }}^{\star}$ when $r=1$

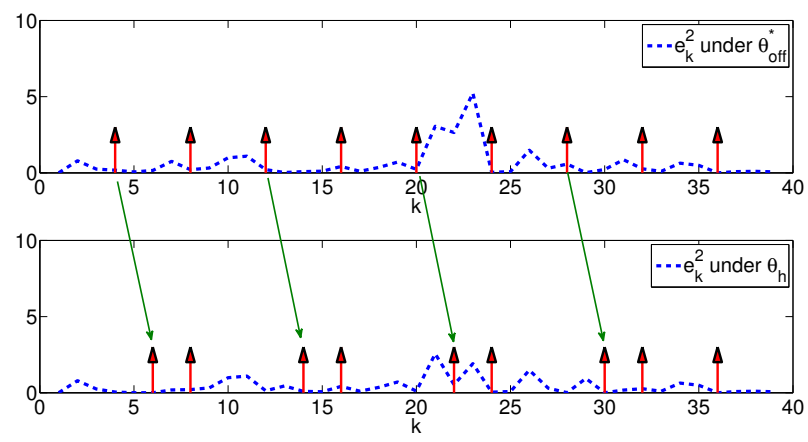

Fig. 4. A sample path of the estimation errors under $\theta_{\mathrm{h}}$ and $\theta_{\text {off }}^{\star}$

\section{EXAMPLES}

\section{A. First-order Systems}

Consider the following parameters for system (1)-(2): $A=$ 1.01, $C=1, Q=R=0.5, m=99, T=399, q=2$. The optimal offline schedule $\theta_{\text {off }}^{\star}$ is periodic with period 4 . The local Kalman filter converges to its steady-sate value, $\bar{P}=$ 0.3101, from which one obtains $h^{2 q}(\bar{P})=2.3969, \Gamma_{0}=$ 1.1264, $\Gamma_{1}=3.1922$ and $\Gamma_{2}=5.3419$. Then we solve for $\delta_{\max }=1.3809$ and $\delta^{\star}=0.7$. Fig. 3 plots $J\left(\theta_{\text {off }}^{\star}\right)$ and $J\left(\theta_{\mathrm{h}}\right)$ for various values of $\delta$. From the figure, $\delta_{\max }$ is 1.38 , and the optimal $\delta$ is 0.7 where the difference between $J\left(\theta_{\mathrm{h}}\right)$ and $J\left(\theta_{\text {off }}^{\star}\right)$ achieves its maximum. The empirical results from Fig. 3 therefore agrees well with Theorem 5.2, and with Theorem 5.4 for $r=1$.

We further plot $e_{k}^{2}$ in a sample path of $\theta_{\text {off }}^{\star}$ and $\theta_{h}$ (taking $\delta=0.7)$ from $k=0$ to $k=39$ in Fig. 4 , where a red arrow indicates a particular time $k$ when $\hat{x}_{k}^{s}$ is sent. Clearly, by rescheduling the transmission at appropriate times (e.g., even transmission instances under $\theta_{\text {off }}^{\star}$ ), the estimation error is reduced. These four instances indicated in the plot demonstrate intuitively why and how the estimation error can be reduced by the proposed hybrid schedule, as one can note that the hybrid schedule allocates these four samples at more suitable times than the offline schedule.

\section{B. Second-order System}

Consider the following system (1)-(2) with parameters: $A=\left[\begin{array}{cc}1 & 0.5 \\ 0 & 1.05\end{array}\right], C=\left[\begin{array}{ll}1 & 0\end{array}\right], Q=$ $\left[\begin{array}{cc}0.5 & 0 \\ 0 & 0.5\end{array}\right], R=0.5, m=49, T=199, q=2$. The optimal offline schedule $\theta_{\text {off }}^{\star}$ is again periodic with period 4 . 


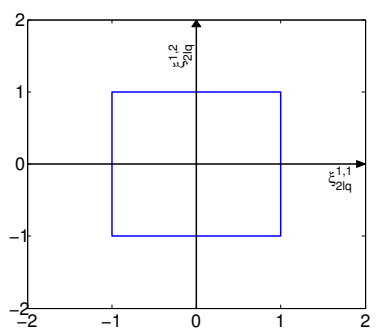

(a)

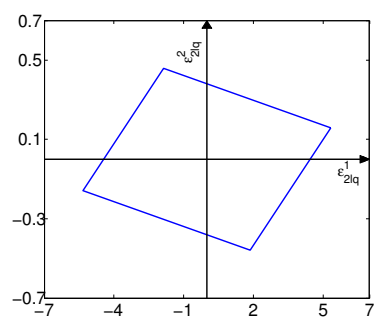

(b)

Fig. 5. The triggering set of $\varepsilon_{2 l q}$ when $r=2$

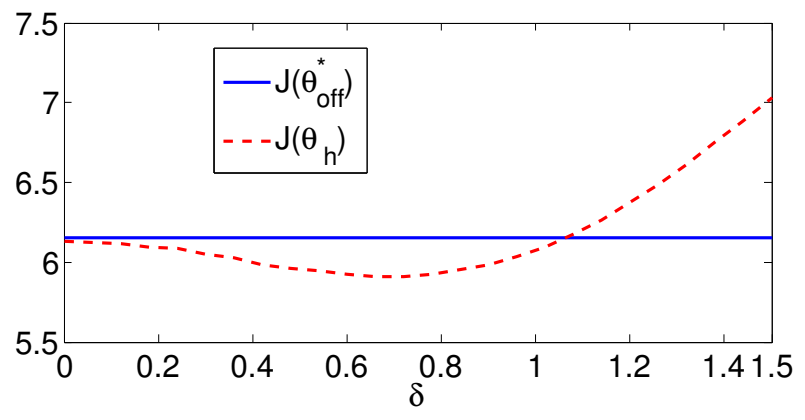

Fig. 6. Comparison of $\theta_{\mathrm{h}}$ and $\theta_{\text {off }}^{\star}$ when $r=2$

The local Kalman filter converges to its steady-sate value

$$
\bar{P}=\left[\begin{array}{ll}
0.3802 & 0.2840 \\
0.2840 & 1.6894
\end{array}\right],
$$

from which one obtains

$$
\begin{gathered}
h^{2 q}(\bar{P})=\left[\begin{array}{cc}
13.3431 & 6.5106 \\
6.5106 & 4.8251
\end{array}\right], \\
\Gamma_{0}=6.0188, \Gamma_{1}=18.5135, \Gamma_{2}=45.5479 \text { and } \\
F=\left[\begin{array}{cc}
-0.2251 & 1.2726 \\
-0.1091 & -2.6253
\end{array}\right] .
\end{gathered}
$$

Given a $\delta$ (e.g., $\delta=1$ ), the event is triggered when $\xi_{k}^{1}$ drop inside the rectangle that is shown in Fig. 5(a), or equivalently $\varepsilon_{2 l q}$ is inside the parallelogram drawn in Fig. 5(b) From part 1) of Theorem 5.2 (with $r=2$ ), we obtain $\delta_{\max }=1.0493$ and from Theorem 5.4, $\delta^{\star}=0.6940$. Fig. 6 plots $J\left(\theta_{\text {off }}^{\star}\right)$ and $J\left(\theta_{\mathrm{h}}\right)$ for different values of $\delta$. This figure shows that $\delta_{\text {max }}$ is about 1.05 and the optimal $\delta$ is approximately 0.7 . Again the empirical results match well with the theoretical ones.

\section{CONCLUSION}

In this paper, we present an improved hybrid sensor schedule to tackle the problem of remote state estimation with limited sensor communications. This schedule leads to better performance when compared with the optimal offline schedule and has a small implementation cost. Future work include extensions to closed-loop control data scheduling and multiple sensor scheduling.

\section{APPENDIX}

The following two lemmas are straightforward to verify and the proofs are omitted.

Lemma 1.1: Let $\mathrm{x} \in \mathbb{R}$ be a Gaussian random variable with zero mean and variance $\mathbb{E}\left[\mathbf{x}^{2}\right]=1$. For $\delta>0$, we have

$$
\mathbb{E}\left[\mathbf{x}^{2}|| \mathbf{x} \mid \leq \delta\right]=1-\beta(\delta)
$$

Lemma 1.2: Let $\xi \in \mathbb{R}^{r}$ be a Gaussian random variable with zero mean and $\mathbb{E}\left[\xi \xi^{\prime}\right]=I_{r}$. Let $\delta \geq 0$. Then

$$
\begin{gathered}
\operatorname{Pr}\left(\|\xi\|_{\infty} \leq \delta\right)=[1-2 Q(\delta)]^{r}, \\
\mathbb{E}\left[\xi \xi^{\prime} \mid\|\xi\|_{\infty} \leq \delta\right]=[1-\beta(\delta)] I_{r} .
\end{gathered}
$$

\section{REFERENCES}

[1] J. Hespanha, P. Naghshtabrizi, and Y. Xu, "A survey of recent results in networked control systems," Proceedings of the IEEE, vol. 95, no. 1, pp. 138-162, 2007.

[2] Y. Mo, L. Shi, R. Ambrosino, and B. Sinopoli, "Network lifetime maximization via sensor selection," in Proceedings of the Asian Control Conference, 2009, pp. 441-446.

[3] R. Ambrosino, B. Sinopoli, and K. Poolla, "Optimal sensor scheduling for remote estimation over wireless sensor networks," Modelling, Estimation and Control of Networked Complex Systems, vol. 1, pp. 127-142, 2009.

[4] V. Gupta, T. Chung, B. Hassibi, and R. M. Murray, "On a stochastic sensor selection algorithm with applications in sensor scheduling and dynamic sensor coverage," Automatica, vol. 42, no. 2, pp. 251-260, 2006.

[5] Y. Chen, Q. Zhao, V. Krishnamurthy, and D. Djonin, "Transmission scheduling for optimizing sensor network lifetime: A stochastic shortest path approach," IEEE Transactions on Signal Processing, vol. 55, no. 5, pp. $2294-2309$, May 2007.

[6] L. Shi, P. Cheng, and J. Chen, "Sensor data scheduling over a packetdropping network," in the 8th World Congress on Intelligent Control and Automation, July 2010, pp. 933-937.

[7] K. J. Åström and B. M. Bernhardsson, "Comparison of Riemann and Lebesgue sampling for first order stochastic systems," in Proceedings of the IEEE Conference on Decision and Control, 2002, pp. 20112016.

[8] O. Imer and T. Başar, "Optimal estimation with limited measurements," in Proceedings of the 44th IEEE Conference on Decision and Control and European Control Conference, December 2005, pp. 1029 -1034 .

[9] L. Li, M. Lemmon, and X. Wang, "Event-triggered state estimation in vector linear processes," in Proceedings of the American Control Conference, July 2010, pp. $2138-2143$.

[10] R. Cogill, "Event-based control using quadratic approximate value functions," in Proceedings of the 48th IEEE Conference on Decision and Control held jointly with the 28th Chinese Control Conference, December 2009, pp. $5883-5888$.

[11] L. Shi, K. H. Johansson, and L. Qiu, "Time and event-based sensor scheduling for networks with limited communication resources," in World Congress of the International Federation of Automatic Control (IFAC), vol. 18, no. 1, 2011, pp. 13263-13268.

[12] B. Anderson and J. Moore, Optimal Filtering. Prentice Hall, 1979. 